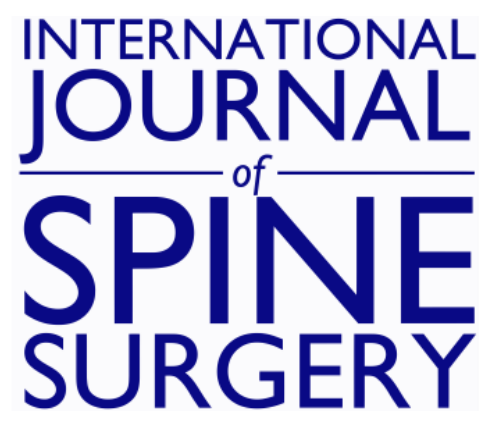

\title{
Distal Ventral Iliac Pathway for Spinopelvic Fixation: Technique Description and Case Series
}

Clarke I. Cady-McCrea, Zachary R. Visco, William F. Lavelle and Richard A. Tallarico

Int J Spine Surg 2021, 15 (5) 988-994

doi: https://doi.org/10.14444/8116

http://ijssurgery.com/content/15/5/988

This information is current as of April 26, 2023.

Email Alerts Receive free email-alerts when new articles cite this article. Sign up at:

http://ijssurgery.com/alerts

The International Journal of Spine Surgery

2397 Waterbury Circle, Suite 1,

Aurora, IL 60504, Phone: +1-630-375-1432 


\title{
Distal Ventral Iliac Pathway for Spinopelvic Fixation: Technique Description and Case Series
}

\author{
CLARKE I. CADY-MCCREA, MD, ZACHARY R. VISCO, MD, WILLIAM F. LAVELLE, MD, RICHARD A. \\ TALLARICO, MD \\ Department of Orthopedic Surgery, SUNY Upstate Medical University, Syracuse, New York
}

\begin{abstract}
Background: Pelvic fixation improves the stability of spinal instrumentation and can be used in high-grade degenerative disease, trauma, deformity, and destabilizing invasive pathologies, such as infection and tumor. Classic techniques for spinopelvic fixation include traditional iliac screws and S2-Alar-Iliac screws. We present a case series describing the distal ventral iliac pathway (DVIP) for spinopelvic fixation and discuss surgical indications and merits of this technique. We describe the use of the DVIP for spinopelvic fixation in the setting of degenerative and traumatic pathologies, compare this technique with existing approaches, and summarize literature to support this approach.

Methods: One hundred twenty-eight cases of DVIP screws were identified at 1 academic medical center, and 3 cases were chosen as representative examples for technique demonstration.

Results: Patient ages ranged from 19 to 81 (mean 62) years. Intraoperative and postoperative complications include 12 incidental durotomies, 3 suprafascial infections, and 2 compressive hematomas. There were 22 instances of hardware failure and 8 instances of pseudoarthrosis. Overall, 26 patients underwent revision surgery. Mean estimated blood loss, operative time, and time under fluoroscopy were $1959 \mathrm{~mL}, 386$ minutes, and 3.19 minutes, respectively.

Conclusions: The DVIP is both safe and effective as a treatment for patients with degenerative and traumatic lumbosacral pathology. Spinopelvic fixation provides improved soft tissue coverage and fewer hardware complications at minimum of 1 year follow up. This case series demonstrates a novel surgical technique for spinopelvic fixation in the setting of numerous spinal pathologies.

Level of Evidence: 4.

Clinical Relevance: This surgical technique is less technically challenging than current approaches, minimizes radiation exposure, and obviates the need for horizontal connector rods. In addition, in highly destabilizing pathologies, this technique also allows for multiple screw placement within the ilium, while maintaining the ability to connect to a single rod construct. This technique is safe, technically approachable, and broadly applicable to an array of spinopelvic pathologies.
\end{abstract}

New Technology

Keywords: spinopelvic fixation, iliac screws, distal ventral iliac pathway (DVIP), posterior superior iliac spine (PSIS)

\section{INTRODUCTION}

Spinopelvic fixation is a useful tool for augmenting the strength and stability of multilevel spinal constructs and play a critical role in treating pathologies which include chronic deformity and traumatic and infectious etiologies. ${ }^{1}$ Construct strength across the lumbosacral junction is particularly important, given the high biomechanical stress at this interval and relatively poor bone quality of the sacrum in certain cases, which predisposes patients to hardware pullout and failure. $^{2}$ Additionally, anatomic constraints limit the size of screws that can be placed into the sacrum, thereby necessitating fixation to the bony pelvis to achieve sufficient construct strength. Since Galves- ton rods first gained popularity in the 1980s, a variety of improvements have been proposed for spinopelvic fixation, most popularly traditional iliac (TI) screws and more recently S2-Alar-Iliac (S2AI) screws. $^{3}$

TI screws are placed at the posterior superior iliac spine (PSIS) and directed to either the superior acetabular notch or the anterior inferior iliac spine (AIIS), with the latter trajectory affording placement of longer screws and mitigating the risk of acetabular violation. ${ }^{4,5}$ Despite a biomechanical advantage over earlier fixation methods, their position most often necessitates horizontal connectors to complete the construct. The positioning of this screw often predisposes the patient to pain and 

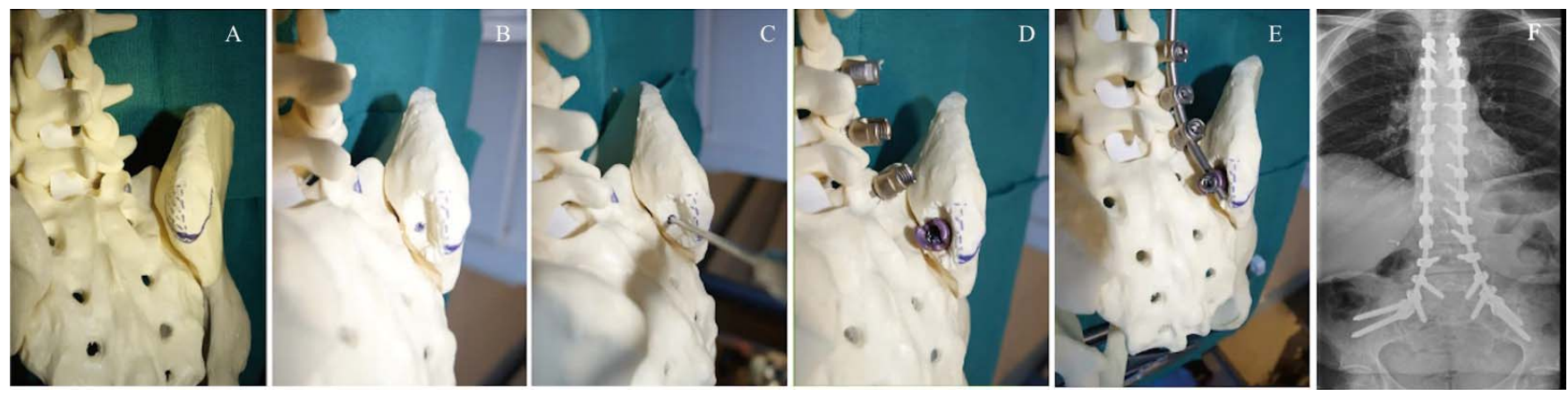

Figure 1. Sawbones model illustrating right distal ventral iliac pathway (DVIP) recess creation and instrumentation. (A)-(E) lliac screws are placed in line with S1 pedicle screws, and the vertical construct is completed with a single rod. (F) The DVIP recess is sufficient for placement of multiple iliac screws bilaterally.

soft tissue complications in association with hardware prominence. ${ }^{3}$ Additionally, offset connectors are a potential point of hardware failure. Lastly, concerns of pain and hardware prominence often necessitate subsequent elective iliac screw removal. In contrast, S2AI screws are placed lateral to the interval between the $\mathrm{S} 1$ and $\mathrm{S} 2$ neural foramina, again aimed at the AIIS. ${ }^{6}$ Since these iliac screws are in line with $\mathrm{S} 1$ and lumbar pedicle screws, this technique obviates the need for lateral connectors. ${ }^{3}$ Furthermore, this technique allows for longer screw placement, theoretically conferring greater construct strength while minimizing hardware prominence and soft tissue disruption. ${ }^{3}$ However, S2AI screws are technically arduous and frequently require extensive radiation exposure to confirm placement. ${ }^{6,7}$ Additionally, the S2AI screws traverse the unfused sacroiliac (SI) joint, which may lead to pain and excess stress on the spinopelvic fixation elements, ultimately leading to screw breakage, ${ }^{8}$ which may necessitate revision surgery for hardware replacement or removal.

We present a novel technique for pelvic fixation with distal ventral iliac pathway (DVIP) screws, supported by the presentation of 3 cases demonstrating the broad utility of this technique. This technique uses a distal, ventral recess on the PSIS for screw placement, allowing for in-line connection to the caudal aspect of the construct, thus obviating the need for offset connectors. This technique maximizes fixation strength, minimizes tissue dissection, and reduces hardware prominence. Furthermore, DVIP screws are placed under direct visualization, making it a more approachable procedure for most spine surgeons and ultimately reduces intraoperative radiation exposure. Surgical considerations and advantages are presented, as well as indications for this procedure.

\section{METHODS}

\section{Data Collection}

A retrospective review of all DVIP screw cases performed by the senior author in patients $\geq 18$ years old from December 2012 to September 2018 was completed. Patients were included in the analysis if they had at least 12 months of follow up and if they had complete operative details in their chart. One hundred twenty-eight cases were identified from our institution's electronic medical record using these criteria. Three representative cases were chosen for this manuscript to demonstrate the utility of this technique. All patients were assessed preoperatively with plains films and spinal computed tomography or spinal magnetic resonance imaging (MRI). All patients were assessed postoperatively with plain films. This retrospective review was declared exempt by the SUNY Upstate Institutional Review Board, which waived the need to obtain informed consent.

\section{Operative Technique}

Patients were placed in the prone position, and posterior dissection was carried out in standard fashion to expose the most cranial level receiving instrumentation, the sacrum caudally, and distal ventral aspect of the PSIS bilaterally, being certain to minimize soft tissue disruption at the fascial insertions. Neuromonitoring was performed intraoperatively with somatosensory and transcortical motor potentials. A rongeur was used to create a recess in the distal ventral half of the PSIS, taking care to leave the osseous roof and its fascial attachments intact. The recess was made deep enough to allow full coverage of the screw head by the PSIS roof (Figure 1). Pilot holes were created in the most distal ventral aspect of the PSIS within the 

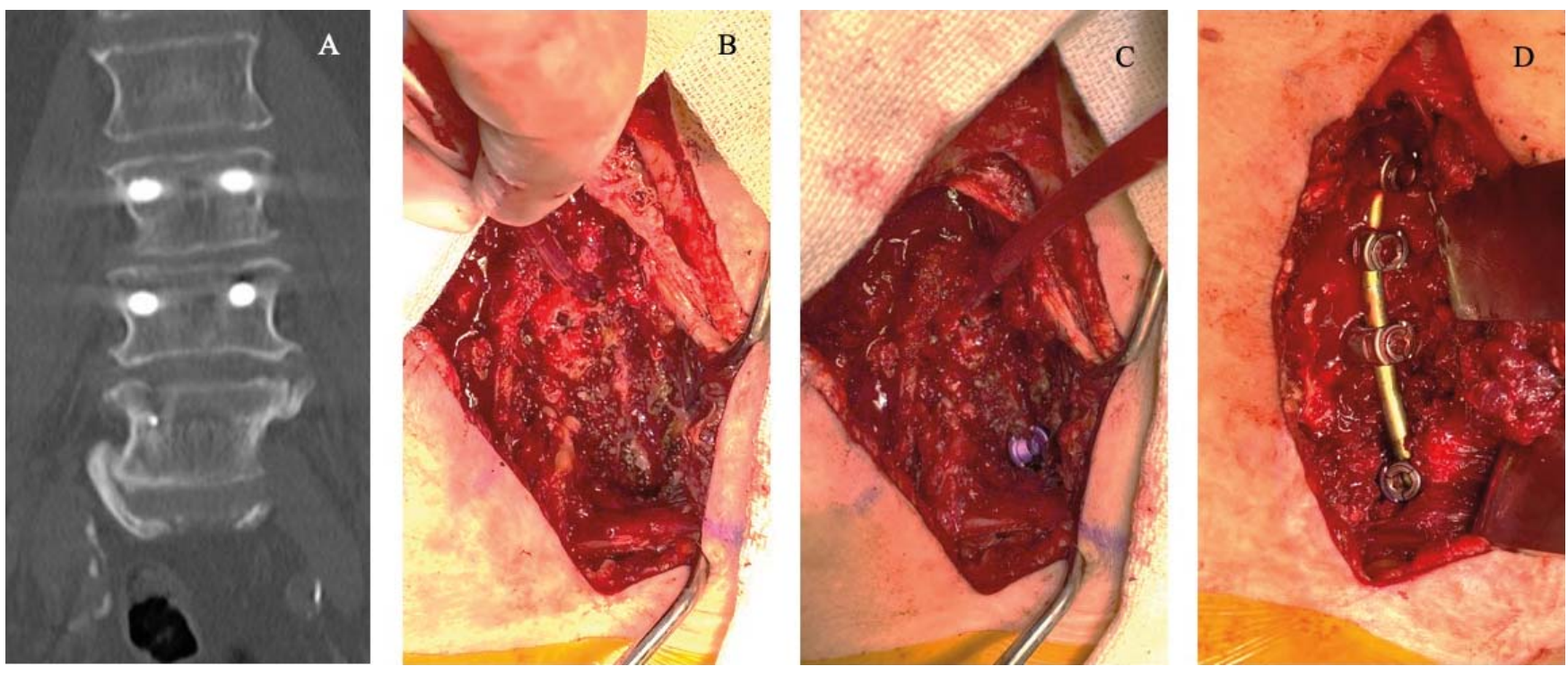

Figure 2. Patient presenting with radicular pain was found to have distal junctional failure of L3-5 posterior instrumentation demonstrating kyphotic collapse postoperatively. Imaging reveals kyphotic collapse associated with vertebral body fracture and fixation failure at L5. Intraoperative photos of lumbosacral deformity treated with revision L3-L5 fusion and extension to the ilium with distal ventral iliac pathway screws.

exposed cancellous bed. The $3.2 \mathrm{AO}$ drill bit trajectory was aimed toward the AIIS in a pathway traversing the dense bone cranial to the sciatic notch. Subsequently, the pilot hole was probed to confirm interosseous position. Iliac screws were then placed along the DVIP and seated such that the tulip heads were contained within the recess below the osseous roof. The most used screw size was 8.5 $\mathrm{mm}$ in diameter and $80 \mathrm{~mm}$ in length. Cranialcaudal biased polyaxial screws were used for all patients in this series. In cases requiring additional points of fixation, multiple screws were placed using the same trajectory in a slightly more cranial position. The remaining construct was completed in standard fashion with spanning rods and bone graft in the posterolateral position (Figure 2). SI joint arthrodesis was performed bilaterally by osteotomizing the sacral and iliac joint surfaces and then placing bone graft over the decorticated beds in most cases. A lumbar drain was placed in cases involving neural decompression, and fascia and skin were closed in standard fashion.

\section{Clinical Presentations}

Case details are summarized in Table 1.

\section{Degenerative Deformity}

Patient $\mathrm{A}$ is a 61-year-old female with a known history of von Willebrand disease and vertebraeanal-trachea-esophagus-renal syndrome who presented with progressive bilateral radicular pain, parasthesias, and gait disturbances that had worsened over the prior 2 years. Her presentation and symptoms were consistent with neurogenic claudication. Imaging revealed degenerative spondylolisthesis, sacral dysgenesis, an L3-L5 vertebral autofusion with kyphotic deformity, anterolisthesis of L2 and retrolisthesis of S1 on the L3-L5 autofusion, and advanced degenerative changes with severe central canal stenosis at L2-L3 (Figure $3)$.

Intraoperatively, desmopressin was administered to mitigate blood loss. Bilateral pedicle screws were placed at L1-L3 and L5, followed by bilateral DVIP screws. No screws were placed in the sacrum due to frankly visible dysgenesis and absence of pedicles. Central decompression was achieved via bilateral laminectomies from L2-S1. Lateral recesses were decompressed by undercutting the medial aspect of the superior articular facet and removing hypertrophic tissue. Bilateral foraminotomies were per-

Table 1. Selected patient and case demographics.

\begin{tabular}{lcclcccc}
\hline Patient & Age (y) & Sex & Surgical Indication & Estimated Blood Loss (mL) & Operative Time (min) & Fluoroscopic Time (s) & Follow Up (mo) \\
\hline A & 61 & F & Spinal deformity & 1000 & 288 & 151.7 & 28 \\
B & 19 & M & Trauma & 750 & 247 & 74.7 & 24 \\
C & 62 & F & Infection & 1700 & 216 & 24.6 \\
\hline
\end{tabular}



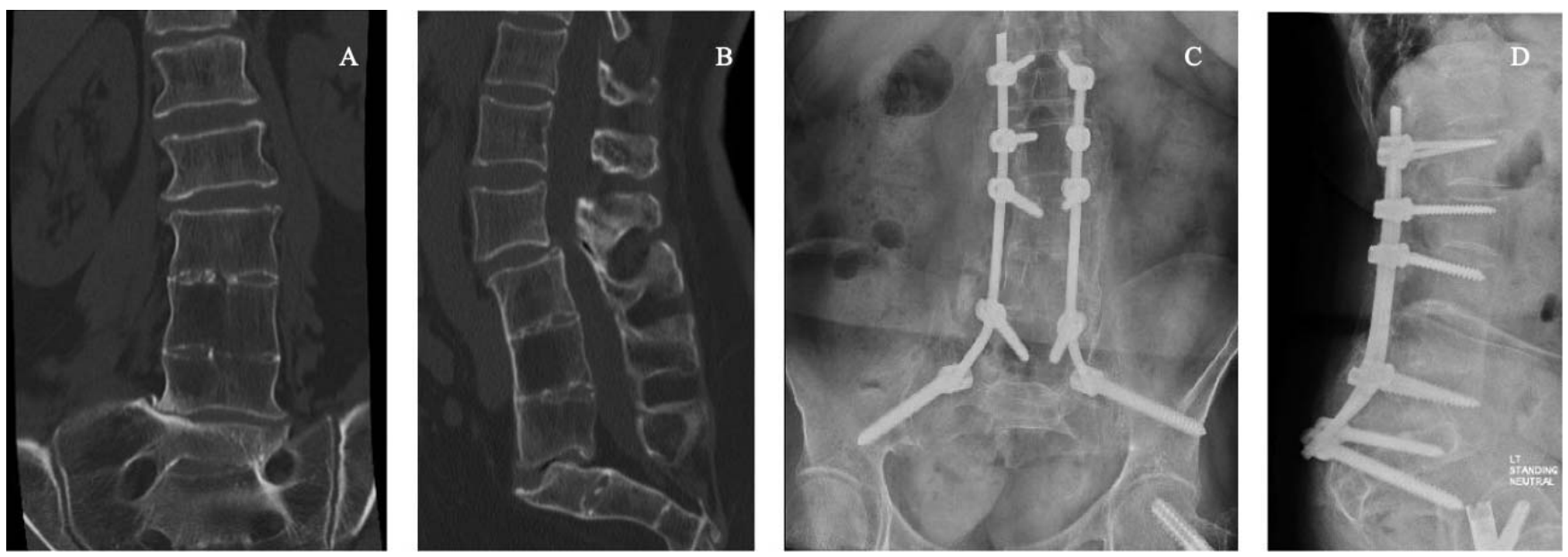

Figure 3. Preoperative and postoperative imaging of lumbosacral deformity treated with L1-ilium fusion with distal ventral iliac pathway screws. (A) and (B) Preoperative sagittal and coronal computed tomography scan of patient with vertebrae-anal-trachea-esophagus-renal syndrome and sagittal imbalance demonstrating autofusion of L3-5 with associated stenosis and listhesis as well as sacral dysgenesis. (C) and (D) Postoperative sagittal and anterior-posterior x-rays at 29 months follow up demonstrating solid fusion with absence of hardware failure.

formed at L5-S1. At the most recent follow up, 29 months postoperatively, there was no evidence of hardware failure, deformity progression, or pseudoarthrosis (Figure 3).

\section{Pelvic Trauma}

Patient B is a 19-year-old male involved in a highspeed motor vehicle collision, resulting in left lateral compression 3 pelvic ring injury with a left displaced comminuted zone 2 sacral fracture, right SI joint dislocation, left segmental superior and inferior ramus fractures, L1-L5 left transverse process fractures, left L5 pedicle fracture, and right S1 pedicle fracture, constituting a form of spinopelvic dissociation.

Given the instability of his sacral fractures, an initial staged procedure was performed by an orthopaedic traumatologist, including reduction and placement of 3 transsacral percutaneous screws. The second stage was performed 6 days later and included spinopelvic fixation. The right-sided S1 pedicle and left-sided L5 fractures precluded bilateral pedicle screws at these levels. Therefore, pedicle screws were placed bilaterally at L4, on the right at L5, and on the left in S1. DVIP screws were placed bilaterally into the ilium, and SI joint arthrodesis was performed at this index procedure. At the most recent follow up, 24 months postoperatively, there was no evidence of hardware failure, deformity progression, or pseudoarthrosis (Figure 4).

\section{Infection}

Patient $\mathrm{C}$ is a 62-year-old female presented to the emergency department with progressive onset fe- vers, axial back pain, and bilateral radiculopathy. MRI revealed increased T2 and short-tau-inversionrecovery signal in the L4 and L5 vertebral bodies consistent with discitis or osteomyelitis. Enhancement was demonstrated throughout the epidural spaces at L4 and L5, causing central canal stenosis abutting the L5 nerve roots. After medical management with intravenous antibiotics, she had worsening back and radicular pain and was found to have increased destruction of the inferior L4 and superior L5 endplates with pathologic kyphotic collapse, resulting in severe central canal stenosis.

Intraoperatively, pedicle screws were placed at L3, L4, and S1, with appropriate screw purchase at L4 despite the fracture. No screws were placed at L5 due to extensive destruction with collapse. DVIP screws were placed bilaterally. Bilateral laminectomies and foraminotomies were performed at L3-S1, along with subtotal facetectomies bilaterally at L4-5 and on the left of L5-S1. All traversing nerve roots were free from compression at completion. A transforaminal lumbar interbody fusion was performed in the posterolateral position at L5-S1 using a titanium cage, given the lytic destruction and inability to instrument L5. At the most recent follow up, 24 months postoperatively, there was no evidence of hardware failure, deformity progression, or pseudoarthrosis (Figure 5).

\section{Patient Outcomes}

One hundred twenty-eight patients with an average follow-up period of 29.5 months were identified from our institution's electronic medical 

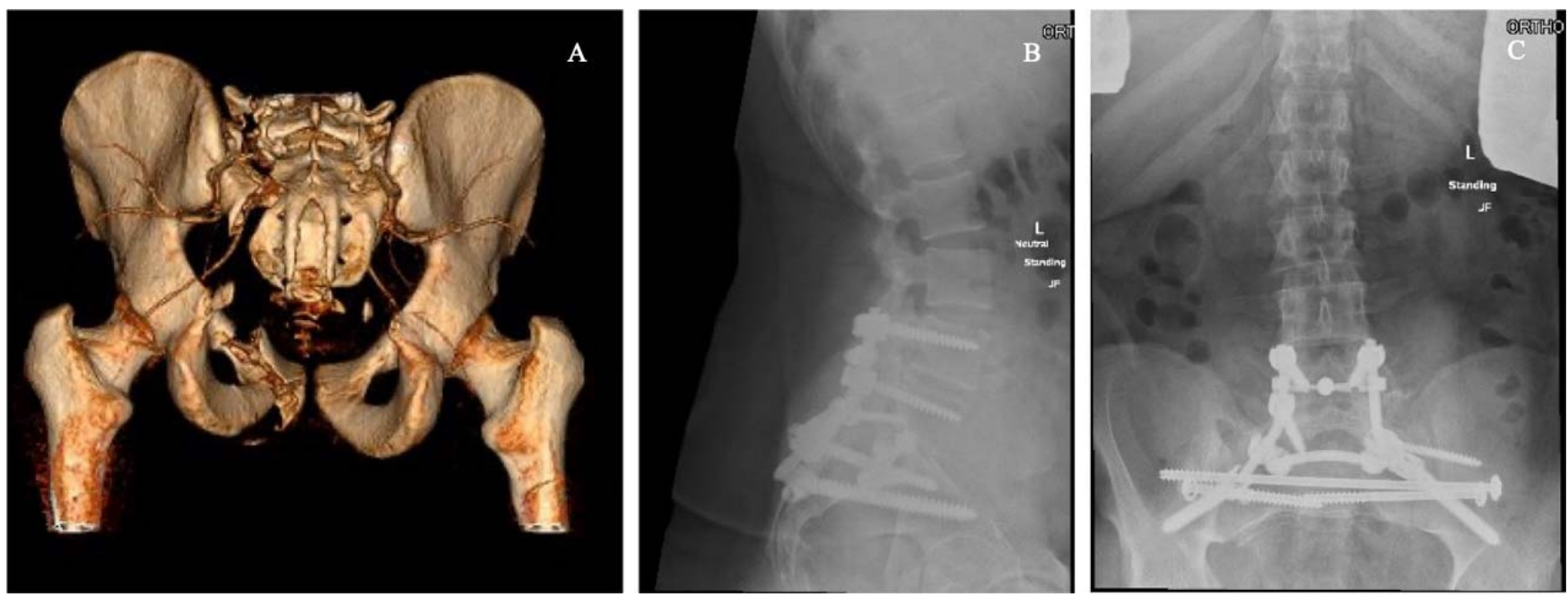

Figure 4. Preoperative and postoperative imaging of spinopelvic trauma treated with L4-ilium fusion with distal ventral iliac pathway screws. (A) Three-dimensional reconstruction of preoperative computed tomography scan demonstrating left lateral compression 3 pelvic ring injury with a left complete displaced comminuted zone 2 sacral fracture, right sacroiliac joint dislocation, left segmental superior and inferior ramus fractures, L1-L5 left transverse process fractures, left L5 pedicle fracture, and right S1 pedicle fracture, constituting a form of spinopelvic dissociation. (B) and (C) Postoperative sagittal and anterior-posterior $\mathrm{x}$-rays at 24 months follow up demonstrating stable fusion without hardware failure.

record and deemed eligible for inclusion in this study. All DVIP screws were placed in the setting of spinal deformity surgery by the senior author. For these procedures, mean estimated blood loss, operative time, and fluoroscopy time were 1959 $\mathrm{mL}, 386$ minutes, and 3.19 minutes, respectively. Intraoperative complications include 12 incidental durotomies, which were repaired without negative sequelae. Three suprafascial infections and 2 compressive hematomas requiring surgical debridement and drainage were identified in the early postoperative period. Late postoperative complications included 22 instances of hardware failure (mean = 17.9 months to radiographic identification). These included 12 rod fractures, 4 DVIP set cap dissociations, 5 proximal screw-rod dissociations, and 1 pedicle screw head fracture. There were no instances of DVIP screw fracture. Twenty-six patients required revision surgery (mean $=22.1$ months postoperatively). Revision surgery was required in 11 patients with hardware failures (mean $=13.8$ months postoperatively). DVIP screws were removed electively in 9 patients due to sacral pain and prominence $($ mean $=26.9$ months postoperatively). Eight patients with documented lumbosacral pseudarthrosis underwent revision surgery (mean = 26.1 months postoperatively). Two patients who
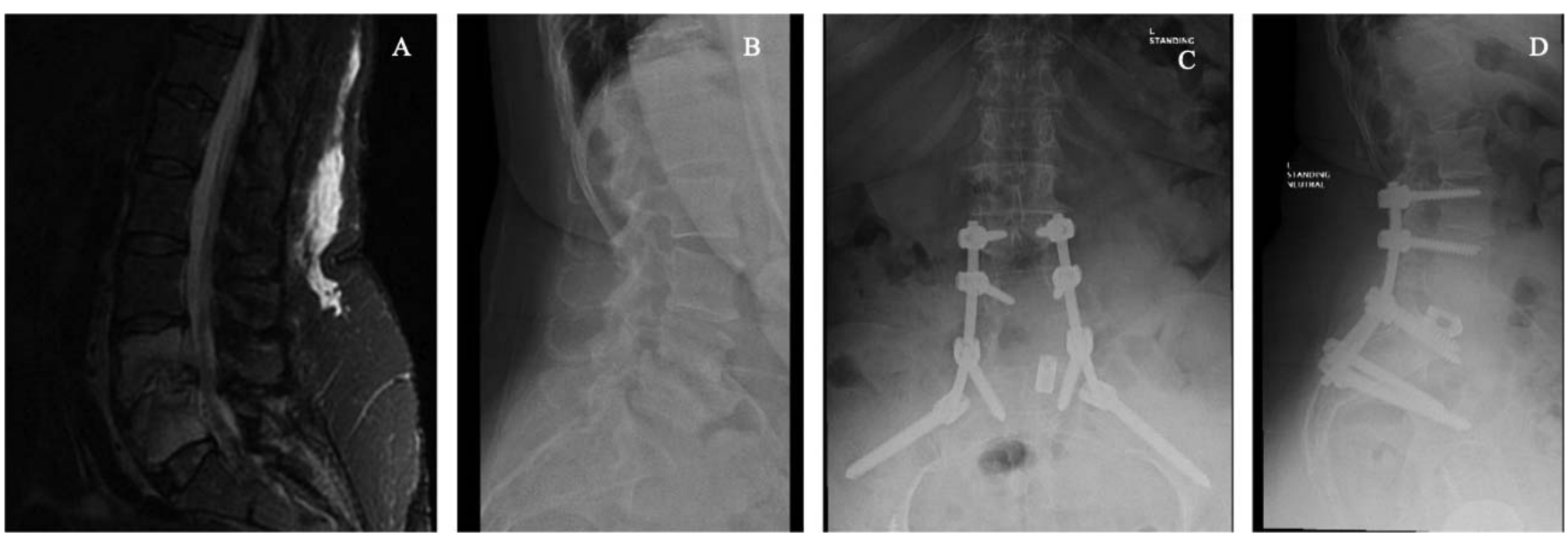

Figure 5. Preoperative and postoperative imaging demonstrating osteomyelitis or discitis treated with L3-ilium fusion using distal ventral iliac pathway screws. Preoperative sagittal magnetic resonance imaging demonstrating osteomyelitis or discitis with infectious destruction of L4-5, epidural enhancement, kyphotic collapse, and central canal stenosis. Preoperative lateral x-ray of the lumbar spine demonstrating pathologic kyphotic collapse. Postoperative anterior-posterior and lateral xrays at 24 months follow up demonstrating solid fusion without hardware failure. 
underwent revision surgery had both lumbosacral pseudoarthrosis and hardware failure.

\section{DISCUSSION}

Given that the lumbosacral junction is a critical spinal motion segment, it is difficult to immobilize during spinal fixation surgeries. ${ }^{9}$ High rates of pseudarthrosis and associated instrumentation failures are well documented, particularly at the sacrum. Spinopelvic fixation, specifically fixation to the ilium, confers improved resistance against the powerful cantilever forces that contribute to these complications, especially in long fusion constructs. ${ }^{9}$ Extending instrumentation past S1 with fixation into the pelvis has been shown to minimize instrumentation failures, reduce pseudoarthrosis rates, and limit the development of flat-back deformity, resulting from cases of distal fixation failure. $^{10-12}$ Increased stiffness at this junction appears to maintain long-term surgical correction in the setting of high grade deformities, making it a useful tool in treating a myriad of pathologies including trauma, infection, and spinal deformity, including sacral fractures, spondylolisthesis, long segment arthrodeses, and chronic degenerative pathologies. ${ }^{2,12,13}$

Numerous methods for pelvic fixation have been well defined in the literature. ${ }^{5,6}$ The seminal Galveston technique uses a contiguous vertical rod that is bent and inserted directly into the ilium, but it lacks resistance to axial strain., ${ }^{9,12}$ This technique predates the adoption of pedicle and iliac screws and lacks solid fixation into cortical bone, which predisposes to pullout from the ilium. ${ }^{12,14}$ As surgical techniques evolved, iliac screws improved upon the Galveston technique by allowing segmental fixation into the ilium. Specifically, iliac screws were placed into the high point of the PSIS. ${ }^{15}$ While this approach provides deep fixation into the ilium at the level of the PSIS, it requires offset connectors to reach the spanning rod and is often complicated by pain and prominent hardware due to poor tissue coverage. ${ }^{2,5}$ By contrast, S2AI screws have a proposed advantage because they minimize screw head prominence, based on a more ventral starting point. ${ }^{5}$ However, these S2AI screws include technically demanding trajectories, necessitating increased fluoroscopy time to confirm safe placement. $^{5}$ Furthermore, this screw trajectory crosses the unfused SI joint, placing these screws at elevated risk for failure in the form of breakage.
The DVIP technique builds upon existing surgical techniques for spinopelvic fixation. Much like the S2AI screws, the DVIP screws allow for placement of a single spanning rod bilaterally, obviating the need for offset connectors. Unlike the S2AI technique, the DVIP technique allows for free-hand placement under direct visualization without the need for fluoroscopy, potentially reducing radiation exposure and operative time. The recess allows the screws to be medialized and placed in line with the vertical construct, fully obviating the need for a horizontal offset connector. Ebata et $\mathrm{al}^{16}$ identified the need for recessed screw placement to ensure adequate tissue coverage of the iliac screw heads. The DVIP technique allows for screws to be recessed into the distal ventral aspect of the PSIS, which prevents the screw heads from protruding above the osseous roof and fascial insertions. Our series demonstrates only $2.3 \%$ of patients suffered wound infections, compared with a reported $25.4 \%$ of patients with TI screws and $2.6 \%$ of patients with S2AI screws in a recent meta-analysis. ${ }^{3}$ Similarly, $7.0 \%(9 / 128)$ of our patients complained of sacral screw pain or prominence, compared with $18.1 \%$ of TI patients and $1.8 \%$ of S2AI patients, further highlighting the benefits of reduced screw prominence. ${ }^{3}$ Furthermore, the broad surface of the ventral PSIS can facilitate multiple screw placement per side in the event that additional spinopelvic fixation is needed, while still allowing a single spanning rod construct (Figure 1). Each additional pathway and its associated screw are placed 1 screw width cranial to the most distal DVIP screw but remain within the distal ventral confines of the PSIS.

Our case series demonstrates that DVIP screws provided stable spinopelvic fixation at long-term follow up, with the absence of DVIP screw fracture supporting arthrodesis of the SI joint. In total, there were 12 intraoperative incidental durotomies, 22 instances of hardware failure, and 26 revision procedures. Thirteen revisions were directly associated with DVIP screw complications, 4 because of set cap dissociation, and 9 DVIP screw removals due to sacral pain and prominence. The durotomies were associated with decompressive procedures in both primary and revision settings, unrelated to DVIP screw placement. Most hardware failures were due to rod failure, commonly seen in conjunction with pseudoarthrosis at the lumbosacral junction. Rod failure in lieu of DVIP screw loosening or breakage indicates sound fixation of 
the DVIP screw at the screw-bone interface. This is further supported by the absence of DVIP screw pullout or loosening in our series. This technique is safe, technically approachable, and broadly applicable to an array of spinopelvic pathologies.

A major limitation of this retrospective study was that it includes only DVIP patients; therefore, no statistical analysis can be performed to compare its clinical efficacy against other spinopelvic fixation methods. Additional studies by this team of researchers are underway to further validate this approach against previously described surgical techniques, by using both biomechanical cadaveric studies and patient-reported outcome data. Despite these limitations, these initial results indicate multiple benefits of using DVIP screws for spinopelvic fixation.

\section{CONCLUSIONS}

Successful treatment of 128 patients with degenerative, traumatic, and infectious lumbosacral pathology demonstrates the safety and efficacy of the DVIP for spinopelvic fixation. Low rates of soft tissue complications and hardware prominence highlight the benefit of recessing screw heads within the distal ventral PSIS, leaving the overlying fascial insertions intact. Similarly, our reported rates of hardware complications and profile of complications observed support the strength of spinopelvic fixation using DVIP screws. Taken together, our series provides a new tool for spinopelvic fixation in the treatment of a variety of spinal pathologies.

\section{REFERENCES}

1. Moshirfar A, Rand FF, Sponseller PD, et al. Pelvic fixation in spine surgery. Historical overview, indications, biomechanical relevance, and current techniques. J Bone Joint Surg Am 2005;87(Suppl 2):89-106. doi:10.2106/JBJS.E.00453

2. Kebaish KM. Sacropelvic fixation: techniques and complications. Spine (Phila Pa 1976). 2010;35(25):2245-2251. doi:10.1097/BRS.0b013e3181f5cfae

3. De la Garza Ramos R, Nakhla J, Sciubba DM, Yassari R. Iliac screw versus S2 Alar-Iliac screw fixation in adults: a metaanalysis. J Neurosurg Spine. 2018;30(2):253-258. doi:10.3171/ 2018.7.SPINE18710

4. Jain A, Brooks JT, Kebbaish KH, Sponseller PD. Sacral alar iliac fixation for spine deformity. JBJS Essent Surg Tech. 2016;6(1):e10. doi:10.2106/JBJS.ST.15.00074

5. Fridley J, Fahim D, Navarro J, Wolinsky JP, Omeis I. Free-hand placement of iliac screws for spinopelvic fixation based on anatomical landmarks: technical note. Int $J$ Spine Surg. 2014;8:3. doi:10.14444/1003

6. Yilmaz E, Abdul-Jabbar A, Tawfik T, et al. S2 Alar-Iliac screw insertion: technical note with pictorial guide. World
Neurosurg. 2018;113:e296-e301. doi:10.1016/j.wneu.2018.02. 009

7. Shillingford JN, Laratta JL, Tan LA, et al. The free-hand technique for S2-Alar-Iliac screw placement: a safe and effective method for sacropelvic fixation in adult spinal deformity. $J$ Bone Joint Surg Am. 2018;100(4):334-342. doi:10.2106/JBJS.17. 00052

8. O'Brien JR, Yu WD, Bhatnagar R, Sponseller P, Kebaish KM. An anatomic study of the S2 iliac technique for lumbopelvic screw placement. Spine (Phila Pa 1976). 2009;34(12):E439-442. doi:10.1097/BRS.0b013e3181a4e3e4

9. Esmende SM, Shah KN, Daniels AH. Spinopelvic fixation. J Am Acad Orthop Surg. 2018;26(11):396-401. doi:10.5435/JAAOS-D-15-00738

10. Kim YJ, Bridwell KH, Lenke LG, Rhim S, Cheh G. Pseudarthrosis in long adult spinal deformity instrumentation and fusion to the sacrum: prevalence and risk factor analysis of 144 cases. Spine (Phila Pa 1976). 2006;31(20):2329-2336. doi:10.1097/01.brs.0000238968.82799.d9

11. Kuklo TR, Bridwell KH, Lewis SJ, et al. Minimum 2year analysis of sacropelvic fixation and L5-S1 fusion using S1 and iliac screws. Spine (Phila Pa 1976). 2001;26(18):1976-1983. doi:10.1097/00007632-200109150-00007

12. Kostuik JP. Spinopelvic fixation. Neurol India. 2005;53(4):483-488. doi:10.4103/0028-3886.22618

13. McCord DH, Cunningham BW, Shono Y, Myers JJ, McAfee PC. Biomechanical analysis of lumbosacral fixation. Spine (Phila Pa 1976). 1992;17(8 Suppl):S235-S243. doi:10. 1097/00007632-199208001-00004

14. Boos N, Webb JK. Pedicle screw fixation in spinal disorders: a European view. Eur Spine J. 1997;6(1):2-18. doi:10. 1007/BF01676569

15. Nguyen JH, Buell TJ, Wang TR, et al. Low rates of complications after spinopelvic fixation with iliac screws in 260 adult patients with a minimum 2-year follow-up. J Neurosurg Spine. 2019;30(5):635-643. doi:10.3171/2018.9.SPINE18239

16. Ebata S, Ohba T, Oba H, Haro H. Bilateral dual iliac screws in spinal deformity correction surgery. J Orthop Surg Res. 2018;13(1):260. doi:10.1186/s13018-018-0969-9

Disclosures and COI: The authors declare no conflicts of interests with this manuscript. This research received no specific grant from any funding agency in public, commercial, or not-for-profit sectors.

Corresponding Author: Richard A. Tallarico, MD, Department of Orthopedic Surgery, SUNY Upstate Medical University, 750 E. Adams St, Syracuse, NY 13210. Phone: (315) 464-8622; Email: tallarir@upstate.edu.

Published 3 December 2021

This manuscript is generously published free of charge by ISASS, the International Society for the Advancement of Spine Surgery. Copyright (c) 2021 ISASS. To see more or order reprints or permissions, see http://ijssurgery.com. 